\title{
La Internacionalización de las Bases de Datos Genéticos: Beneficios y Riesgos
}

Internationalization of genetic databases: Benefits and Risks

\section{Inmaculada Vivas Tesón ${ }^{1}$}

RESUMEN: La información genética en las bases de datos están en constante crecimiento. La defensa de la opinión de que el contenido de estas bases debería estar a disposición de científicos de todo el mundo, tiene riesgos y beneficios. Este artículo tiene como alcance trazar la escena internacional esta investigación genómica y la creación de un espacio virtual centralizado de la información genética con el único propósito de la investigación y las implicaciones legales de los datos personales de los pacientes.

Palabras clave: Genoma, internacionalización, enfermedades

RESUMO: As informações genéticas em bases de dados estão em constante crescimento. A defesa de opinião de que o conteúdo dessas bases devem estar disponíveis para os cientistas de todo o mundo, apresenta riscos e benefícios. Este artigo tem como escopo traçar o cenário internacional dessa investigação genômica e a criação de um espaço virtual centralizado de informação genética com fins exclusivos de pesquisa e as implicações jurídicas dos dados pessoais dos pacientes.

Palavras-chave: Genoma, internacionalização, enfermidades.

ABSTRACT: The genetic information in databases are constantly growing. The defense of the view that the content of these bases should be available to scientists from all over the world has risks and benefits. This article has as its scope the goal to trace in the international scene this genomics research and the creation of a centralized virtual space of genetic information with the sole purpose of research and legal implications of personal data of patients.

Keywords: Genome, internationalization, diseases

\section{Introducción}

Todos estamos de acuerdo en que ojalá el cáncer, la diabetes o el Alzheimer tuvieran lo antes posible una solución (jdaríamos lo que fuera!).

Somos conscientes de que, en estos momentos, existe un gran número de investigadores en distintos rincones del mundo secuenciando genomas de personas para investigar sobre éstas y otras muchas enfermedades.

\footnotetext{
${ }^{1}$ Profesora Titular de Universidad de Sevilla. Facultad de Derecho. Sevilla, España. E-mail: ivivas@us.es
} 
Los centros investigadores con capacidad para estudiar los genes humanos se han multiplicado a un ritmo vertiginoso y, en consecuencia, también los estudios y los datos genéticos, además de los notables avances en bioinformática.

Hoy día, existe un universo de posibilidades para la investigación genómica, pero tal vez se trate de un universo disperso y desaprovechado científicamente.

En la actualidad, existen bases de datos genéticos con fines de investigación de diversos tipos (de titularidad privada y pública; de acceso público y restringido), dimensiones y objetivos que contienen información muy valiosa para que la comunidad científica internacional realice estudios genéticos y análisis comparativos, pero también muy dispersa.

El ingente volumen de información genética almacenada en las bases de datos están superando las previsiones, lo que unido a los enormes avances en el campo de la medicina personalizada y preventiva (la farmacogenética), quizás nos deba llevar a plantearnos, en el momento actual, un salto cualitativo en un contexto científico nuevo, en un escenario globalizado.

Un paso indudablemente importante fue la Ley 14/2007, de 3 de julio, de Investigación Biomédica, la cual permite el almacenamiento de datos genéticos de carácter personal ${ }^{2}$, pero ahora nos planteamos si es posible subir un peldaño más: ¿es posible integrar toda la información genética almacenada en las distintas bases de datos haciéndola útil y accesible a la comunidad científica internacional?

La respuesta suscita cuestiones éticas y jurídicas que resolver. Por razones evidentes, centraremos nuestra atención en las segundas.

\section{La Creación de un Espacio Virtual Centralizado de Información Genética con Fines de Investigación}

Ante todo, es preciso advertir que, de un lado, se afrontarán en las próximas páginas aspectos relativos a la gestión de la información genética, no, por tanto, a la problemática que rodea a la dimensión física de la muestra biológica y, de otro, que

\footnotetext{
${ }^{2}$ La LIB define, en su art. 3.j), el „dato genético de carácter personal" como aquélla información sobre las características hereditarias de una persona, identificada o identificable obtenida por análisis de ácidos nucleicos u otros análisis científicos.
} 
nuestra atención se centrará en las bases de datos genéticos con fines de investigación biomédica (art.18, ley 11/2007) y, por consiguiente, no en aquéllas con finalidades sanitarias ni forenses o judiciales (ley orgánica 10/2007).

Claro lo anterior, el interrogante que formulamos es el siguiente: ¿por qué no armonizar los procedimientos de depósito, gestión y acceso a los datos genéticos de las distintas bases o repositorios de centros, proyectos investigadores con el fin de centralizar dicha información y compartirla con la comunidad científica mundial? ¿Por qué no interconectamos las distintas bases de datos o las integramos en una única plataforma virtual 0 nube $^{3}$ a nivel estatal o internacional que contenga toda la información genética?

Dicho de otro modo, ¿no sería útil acceder por internet a una base de datos genéticos con un buscador especializado que permitiera a los investigadores de cualquier parte del mundo, con su correspondiente licencia de uso, realizar consultas de ADN para desarrollar sus experimentos comparando cientos o miles de genomas?

La interconexión o integración de bases de datos puede ser clave para la investigación científica. Sería muy útil estrechar lazos y aunar esfuerzos y, muy especialmente, recursos materiales y humanos ${ }^{4}$.

Es evidente que los beneficios del uso compartido de datos genéticos son innegables, pero si bien cuando se trata de hacer secuencias genéticas de animales o de plantas dicha interconexión o integración de base de datos no plantearía problemas jurídicos, no puede decirse lo mismo cuando se trata de personas.

Cuando se trata de información genética humana comienzan los interrogantes y las dudas desde la óptica jurídica. En relación a los seres humanos, no todo está permitido bajo el argumento de la libertad de la ciencia e investigación o del "interés humanitario"(1)

La cuestión se centra en identificar las libertades, derechos e intereses en juego y dignos de tutela por el Derecho, así como realizar un balance equilibrado de los mismo. A cuestiones reales nuevas (como la que aquí se plantea), soluciones jurídicas nuevas.

\footnotetext{
${ }^{3}$ Ya en 1998, el Parlamento islandés aprobó una ley que permitía la creación de una base de datos centralizada, con información genealógica, genética y médica individual de todos los ciudadanos de Islandia. Asimismo, podemos citar el Proyecto 1000 Genomas, Google Genomics, puesto en marcha en marzo de 2014 o el Archivo Europeo del Genoma y Fenoma (EGA).

${ }^{4}$ El art. 88 LIB contempla la colaboración del Sistema Nacional de Salud con otras instituciones y organizaciones implicadas en la investigación para la utilización conjunta de infraestructuras científicas y el desarrollo de proyectos de investigación.
} 
Es indudable que el Derecho debe acompañar a la Ciencia (eminentemente dinámica o cambiante y sin fronteras) para permitir que avance, si bien, dada la rapidez con la que acontecen sus progresos, el Derecho, en este ámbito, se queda necesariamente atrás; Derecho y Ciencia caminan a dos velocidades distintas. Por ello, tal vez, en estos últimos años, más que crearse figuras jurídicas, las ya existentes se han ido amoldando a las nuevas realidades. La elasticidad del Derecho es más que evidente: piénsese en el valor e importancia que han alcanzado el derecho a la autodeterminación, a la intimidad y a la confidencialidad (se habla de una privacidad genética) y, en consecuencia, la relevancia que reviste, hoy día, el consentimiento informado; el valor de la dignidad humana; la no discriminación por razones genéticas; existe un nuevo concepto de familia, la "familia genética" 5 (del que no forman parte el cónyuge ni los hijos adoptivos y que, en cambio, integran personas extrañas como los donantes de gametos) y, en consecuencia, se alude a un parentesco y línea genéticos.

El Derecho resuelve problemas y trata de eliminar, en lo posible, obstáculos, pero existen principios y límites que respetar y que, por tanto, son infranqueables. Todos ellos, en el ámbito biomédico, pueden simplificarse en uno: el respeto a la dignidad humana (DECLARACIÓN SOBRE GENOMA HUMANO DEL COMITÉ INTERNACIONAL DE BIOÉTICA - CIB, DE LA UNESCO).

Así las cosas, la cuestión aquí planteada podría formularse del siguiente modo: ¿es posible un intercambio responsable, seguro y eficaz de la información genética a nivel mundial entre las distintas comunidades de investigación, cumpliendo con los más altos estándares de privacidad y respeto a la dignidad humana de la persona?

La cuestión aquí planteada, el uso compartido de la información genética, trasciende la libre elección individual y la actividad legislativa nacional, pasando a adquirir una dimensión jurídico-política internacional en la cual ha de marcarse, con suma nitidez, unos mínimos de lo que está permitido, prohibido y es obligatorio.

\footnotetext{
${ }^{5}$ Según el art. 1 de la Recomendación n. R (97) 5, de 13 de febrero de 1997, del Comité de Ministros del Consejo de Europa a los Estados miembros, sobre la protección de datos médicos, "la expresión "datos genéticos" se refiere a todos los datos, cualquiera que sea su clase, relativos a las características hereditarias de un individuo o al patrón hereditario de tales características dentro de un grupo de individuos emparentados".
} 
Vayamos por partes.

\section{Las Singularidades del Dato Genético de Carácter Personal}

\section{Sus implicaciones más allá de la persona}

El dato genético humano, definido por la Declaración Internacional de la UNESCO sobre los Datos Genéticos Humanos de 16 de octubre de 2003, como "información sobre las características hereditarias de las personas, obtenida por análisis de ácidos nucleicos u otros análisis científicos" (ART. 2.I) presenta unas características que lo singularizan al identificar el carácter único de cada individuo, puesto que el código genético de cada persona es exclusivo, pero también puede proporcionar información sobre otros sujetos (Art. 4 DE LA DECLARACIÓN INTERNACIONAL DE LA UNESCO SOBRE LOS DATOS GENÉTICOS HUMANOS) y, por lo tanto, afectar a derechos e intereses no sólo individuales sino también de terceros.

De este modo, la singular información genética de los sujetos trasciende su individualidad para afectar a la familia y a la sociedad y es por ello por lo que es especialmente sensible y requiere un alto nivel de seguridad (LEY ORGÁNICA 15/1999).

Así las cosas, es curioso cómo la libertad de un individuo a participar en una investigación puede entrar en conflicto con la de otro individuo o de toda una comunidad a no participar, ya que las consecuencias de los estudios genéticos pueden llegar a repercutir (positiva o negativamente) en otras personas o grupos. Por tanto, existe un principio de doble protección: del individuo y del grupo o comunidad.

Además, la información genética puede ser relevante (biológica y culturalmente) con posterioridad a la obtención del material biológico, pues los descubrimientos son absolutamente impredecibles en dicho momento, de manera que la posibilidad de informar ex ante a los sujetos sobre el uso a futuro de las muestras y datos asociados es limitada.

Por todo ello, el dato personal genético, al que, como tal, se le deben aplicar los principios y garantías de la legislación de protección de datos (Art. 5.1 LIB), presenta particularidades respecto a los derechos de su titular, su tratamiento y sus usos y finalidades. Tiene implicaciones individuales o colectivas, positivas 0 negativas. 


\section{2.- Privacidad y riesgos de la 'nube genética'}

Dada la peculiar naturaleza de la información genética, la privacidad es una de las cuestiones cruciales en esta materia.

Resulta clave el art. 14 de la ya citada Declaración Internacional de Datos Genéticos Humanos:

a) Los Estados deberían esforzarse por proteger la privacidad de las personas y la confidencialidad de los datos genéticos humanos asociados con una persona, una familia o, en su caso, un grupo identificables, de conformidad con el Derecho interno compatible con el Derecho internacional relativo a los derechos humanos.

b) Los datos genéticos humanos, los datos proteómicos humanos y las muestras biológicas asociados con una persona identificable no deberían ser dados a conocer ni puestos a disposición de terceros, en particular de empleadores, compañías de seguros, establecimientos de enseñanza y familiares de la persona en cuestión, salvo por una razón importante de interés público en los restringidos casos previstos en el Derecho interno compatible con el Derecho internacional relativo a los derechos humanos o cuando se haya obtenido el consentimiento previo, libre, informado y expreso de esa persona, siempre que éste sea conforme al Derecho interno y al Derecho internacional relativo a los derechos humanos. Debería protegerse la privacidad de toda persona que participe en un estudio en que se utilicen datos genéticos humanos, datos proteómicos humanos o muestras biológicas, y esos datos deberían revestir carácter confidencial.

c) Por regla general, los datos genéticos humanos, datos proteómicos humanos y muestras biológicas obtenidos con fines de investigación científica no deberían estar asociados con una persona identificable. Aun cuando estén disociados de la identidad de una persona, deberían adoptarse las precauciones necesarias para garantizar la seguridad de esos datos o esas muestras biológicas.

d) Los datos genéticos humanos, datos proteómicos humanos y muestras biológicas obtenidos con fines de investigación médica y científica sólo podrán seguir estando asociados con una persona identificable cuando ello sea necesario para llevar a cabo la investigación, y a condición de que la privacidad de la persona y la confidencialidad de los datos o las muestras biológicas en cuestión queden protegidas con arreglo al Derecho interno. 
e)Los datos genéticos humanos y los datos proteómicos humanos no deberían conservarse de manera tal que sea posible identificar a la persona a quien correspondan por más tiempo del necesario para cumplir los fines con los que fueron recolectados o ulteriormente tratados".

Así las cosas, ¿qué tipo de datos podrían incluirse en la "nube genética": datos asociados $^{6}$, datos disociados ${ }^{7}$ o datos irreversiblemente disociados de una persona identificable ${ }^{8}$ ?

Si lo que nos planteamos es una especie de Red global de almacenamiento y uso del ADN con fines de investigación, debemos inclinarnos por datos genéticos anonimizados $^{9}$ (la Declaración contempla que, para tales fines, "por regla general", "ho deberían estar asociados con una persona identificable" - art. 14.c), sin embargo, a continuación, permite que excepcionalmente puedan seguir estando asociados con una persona identificable "cuando ello sea necesario para llevar a cabo la investigación, y a condición de que la privacidad de la persona y la confidencialidad de los datos o las muestras biológicas en cuestión queden protegidas con arreglo al Derecho interno" -art. 14.d)-).

De este modo, la anonimización permitiría el libre uso de los datos genéticos, de modo que el sujeto fuente no sufriría ningún perjuicio. Sin embargo, es importante subrayar que existen problemas derivados de la dificultad de lograr una disociación verdaderamente irreversible de la persona de que se trate. El anonimato no está completamente garantizado, ya que es posible, con facilidad, re-identificar a la persona de forma indirecta mediante la combinación de la información genética con otros datos de dominio público (p. ej. accesibles mediante internet).

\footnotetext{
${ }^{6}$ Según los define la Declaración Internacional de Datos Genéticos Humanos, son aquellos datos que contienen información como el nombre, la fecha de nacimiento y la dirección, gracias a la cual es posible identificar a la persona a la que se refieren (art. 2, ix).

${ }^{7}$ Definidos por la Declaración Internacional de Datos Genéticos Humanos como aquellos datos no asociados con una persona identificable por haberse sustituido o desligado toda la información que identifica a esa persona utilizando un código (art. 2,x).

${ }^{8}$ Conforme a la Declaración Internacional de Datos Genéticos Humanos, son aquellos datos que no pueden asociarse con una persona identificable por haberse destruido el nexo con toda información que identifique a quien suministró la muestra (art. 2,xi).

${ }^{9}$ Según el art. 3 de la LIB, letra h) "Dato anónimo" es el dato registrado sin un nexo con una persona identificada o identificable y en su letra i) define el "Dato anonimizado o irreversiblemente disociado" como aquél que no puede asociarse a una persona identificada o identificable por haberse destruido el nexo con toda información que identifique al sujeto, o porque dicha asociación exige un esfuerzo no razonable, entendiendo por tal el empleo de una cantidad de tiempo, gastos y trabajo desproporcionados.

El art. 50.2 LIB dispone: "los datos genéticos de carácter personal sólo podrán ser utilizados con fines epidemiológicos, de salud pública, de investigación o de docencia cuando el sujeto interesado haya prestado expresamente su consentimiento, o cuando dichos datos hayan sido previamente anonimizados" $\mathrm{y}$, a continuación, en su apartado $3^{\circ}$, contempla la excepción de que los datos genéticos puedan ser codificados.
} 
Existen brechas de seguridad y dado, por tanto, que la persona se hace absolutamente vulnerable, exponiéndose a un "desnudo genético integral"10 en la red (y no sólo ella, sino también otras personas), el procedimiento para la prestación del consentimiento informado debería contemplar mecanismos de información precisa y clara acerca de la trascendencia del acto que la persona va a consentir y de los riesgos que está dispuesta a asumir, debiéndose comprobar (tal vez a través de un cuestionario) que dicha información así como la relevancia, significado y el valor del consentimiento han sido efectivamente comprendidas ${ }^{11}$. Dicho de otro modo, el consentimiento informado no puede convertirse en un mero trámite burocrático, sino que ha de tratarse de un consentimiento realmente informado y, si es posible, para evitar recabarlo cada vez que exista una acción científica nueva y las dificultades que encierra el recontactar al sujeto fuente, que legitime ab initio la práctica de todas aquellas operaciones imprevisibles a la hora de la obtención de la muestra biológica, esto es, que se trate de un consentimiento general.

$Y$ el consentimiento informado para que mis datos genéticos sean utilizados para fines de investigación, ¿de he prestarlo yo o todas las personas a las que pudiera afectar algún estudio genético y, eventualmente, resultar estigmatizadas por algún descubrimiento? ¿sería necesario el permiso previo de los demás familiares? ${ }^{12}$

Dado que el consentimiento informado podría tener repercusiones colaterales, ¿cómo se respeta el derecho a no saber de otros familiares, quienes optan por ello en aras de su tranquilidad o equilibrio psíquicos? El consentimiento informado, ¿deja de tener carácter estrictamente individual?

¿Y qué ocurre con el consentimiento a que se compartan los datos genéticos de menores o incapacitados judicialmente?

Por otra parte, podría producirse una discriminación genética en el ámbito laboral (con consecuencias permanentes, no temporales) o en el de seguros de

\footnotetext{
${ }^{10}$ La gráfica expresión no es nuestra sino corresponde al titular de una noticia publicada en el Diario El País por Javier Sampedro el 17 de enero de 2013. Disponible en: http://sociedad.elpais.com/sociedad/2013/01/17/actualidad/1358445379_181975.html.

${ }^{11}$ Interesante resulta el trabajo de ALLEN, J./MCNAMARA, B.: "Reconsidering the value of consent in biobank research", en Biotehics, 2011 Mar;25(3):155-66. doi: 10.1111/j.1467-8519.2009.01749.x., en el que los autores realizan un estudio a través de entrevistas a donantes de muestras biológicas para un estudio sobre el cáncer en Australia se comprueba si efectivamente entendieron el significado de la investigación y del consentimiento.

${ }^{12}$ Conforme a lo dispuesto por el art. 5.2, párrafo $2^{a}$ de la LIB, la respuesta debe ser afirmativa: "en el supuesto de que los datos obtenidos del sujeto fuente pudieran revelar información de carácter personal de sus familiares, la cesión a terceros requerirá el consentimiento expreso y escrito de todos los interesados".
} 
salud $^{13}$. Al respecto, la Carta de Derechos Fundamentales de la UE de 7 de diciembre de 2000, en su art. $21.1^{14}$, prohíbe toda discriminación, en particular, por razón de características genéticas. En igual sentido se pronuncia la Declaración Internacional sobre los datos genéticos humanos de 2003, en su art. 7 a) ${ }^{15}$ y el art. 6 LIB. Por tanto, nadie puede ser estigmatizado por razones genéticas. Existe la dignidad de la persona, con independencia de su genotipo ${ }^{16}$.

¿Y si se llegara a crearse un ADN sintético e, intencionadamente, apareciera en la escena de un crimen?

Otra cuestión a tener en cuenta: la bioseguridad. ¿Con qué medidas de seguridad es almacenada la información genética? ¿y si hay un robo de datos para su más que probable ulterior comercialización? ${ }^{17}$

Necesitamos que nuestros datos genéticos, ya sea por su valor preciado, ya sea por su eventual nocividad, estén contenidos y custodiados en una auténtica caja fuerte con extremas medidas de seguridad ${ }^{18}$. La puesta a disposición de nuestros datos genéticos en la red podría implicar la pérdida de nuestro yo (en este punto, habría que preguntarse: ¿daríamos lo que fuera, incluso nuestro "yo"?).

\footnotetext{
${ }^{13}$ A la discriminación genética hace mención la Declaración Universal sobre el Genoma y Derechos Humanos del 11 de noviembre de 1997 (art. 6); la Declaración Internacional sobre los Datos Genéticos Humanos del 16 de octubre de 2003 (art. 7) y la Declaración universal sobre Bioética y Derechos Humanos de19 de octubre de 2005 (art. 11).

${ }_{4}$ Art. 21.1: "Se prohíbe toda discriminación, y en particular la ejercida por razón de sexo, raza, color, orígenes étnicos o sociales, características genéticas, lengua, $r$ eligión o convicciones, opiniones políticas o de cualquier otro tipo, pertenencia a una minoría nacional, patrimonio, nacimiento, discapacidad, edad u orientación sexual".

${ }^{15}$ Art. 7.a) de la Declaración Internacional sobre los datos genéticos humanos de 2003: "debería hacerse todo lo posible por garantizar que los datos genéticos humanos y los datos proteómicos humanos no se utilicen con fines que discriminen, al tener por objeto o consecuencia la violación de los derechos humanos, las libertades fundamentales o la dignidad humana de una persona, o que provoquen la estigmatización de una persona, una familia, un grupo o comunidades".

${ }^{16} \mathrm{El}$ art. 2: "(a) Cada individuo tiene derecho al respeto de su dignidad y derechos, cualesquiera que sean sus características genéticas", y el artículo 6 de la Declaración de la UNESCO Declaración universal sobre el Genoma Humano y los Derechos Humanos que fue aprobada en Asamblea de la UNESCO, el 11 de noviembre de 1997, establece que "nadie podrá ser objeto de discriminaciones fundadas en sus características genéticas, cuyo objeto o efecto sería atentar contra sus derechos humanos y libertades fundamentales y el reconocimiento de su dignidad“.

17 Desde el punto de vista de la responsabilidad penal, es preciso diferenciar dos conductas delictivas: la del investigador/médico que, teniendo autorización para acceder a la base de datos no anonimizada, se apodera de los datos contenidos en la misma y los utiliza para fines distintos a la investigación médica que está realizando; y la del investigador que, sin autorización alguna, accede a una base de datos. Una apropiación no consentida de las bases de datos biogenéticas para la investigación constituirá un delito de espionaje informático (art. 278 CP); y el apoderamiento, acceso utilización o modificación de datos no anonimizados para la investigación contenidos en una base es un delito de descubrimiento y revelación de secretos que afecta a la intimidad y a la identidad informática (art. $197 \mathrm{CP})$.

${ }^{18}$ La gráfica expresión es de MACILOTTI, M./IZZO, U./PASCUZZI, G./BARBARESCHI, M.: "La disciplina giuridica delle biobanche", en Pathologica, 2008, pág. 86.
} 


\title{
1.3 El Uso Compartido de Datos Genéticos: Su Cobertura Normativa
}

\author{
En el ámbito de la biomedicina, nos encontramos con una gran variedad
} normativa, pues contamos con regulaciones internacionales ${ }^{19}$, europeas ${ }^{20}$, nacionales (si bien son pocos los países que cuentan con regulación propia) y, en nuestro caso, autonómicas. Un verdadero puzzle normativo ${ }^{21}$.

Además, ha de tenerse en cuenta la existencia de otros documentos que, pese a su importancia en el impulso de la actividad legislativa, carecen de fuerza vinculante (recomendaciones, códigos de conducta, etc.), así como el amplio papel concedido a los Comités de ética. En dicho rompecabezas encontramos disposiciones normativas que contemplan el derecho de la persona a participar en el progreso científico ${ }^{22}$, la libertad científica $^{23}$, la circulación transfronteriza de datos genéticos humanos y la cooperación internacional al respecto ${ }^{24}$, el uso compartido de datos ${ }^{25}$, así como el

\footnotetext{
${ }^{19}$ Entre otras, Declaración de Helsinki de 1964; Convenio de Oviedo de 1997; la Declaración Internacional sobre los Datos Genéticos Humanos del 16 de octubre de 2003 y la Declaración universal sobre Bioética y Derechos Humanos de19 de octubre de 2005.

${ }^{20}$ Carta Europea de Derechos fundamentales de 2000.

${ }^{21}$ Para AZZINI, S.: "Biobanche, consenso e fonti del Diritto: un caso si eccezionale disordine", en La disciplina delle biobanche ai fini terapeutici e di ricerca, en CASONATO, PICIOCCHI y VARONESI, 2012, Università di Trento, pág. (http://eprints.biblio.unitn.it/3979/1/casonato piciocchi veronesi quaderno 101 versione corretta giov19apr2012. pdf), "l"analisi delle odierne fonti del diritto e del modo in cui queste interagiscono tra loro dimostra che la situazione della regolamentazione giuridica delle biobanche, con particolare riferimento al consenso informato, non rappresenta un caso di eccezionale disordine delle fonti normative ma, al contrario, è un esempio di tale situazione".

${ }^{22}$ Art. 27 de la Declaración Universal de los Derechos Humanos de 1948.

${ }^{23}$ Art. 15 de Pacto Internacional de Derechos Económicos, Sociales y Culturales de 1966.

${ }^{24}$ Art. 15 de Pacto Internacional de Derechos Económicos, Sociales y Culturales de 1966.

Art. 18 de la Declaración Internacional de la UNESCO de datos genéticos humanos: "Circulación y cooperación internacional

a) De conformidad con su Derecho interno y con los Acuerdos internacionales, los Estados deberían regular la circulación transfronteriza de datos genéticos humanos, datos proteómicos humanos y muestras biológicas para fomentar la cooperación médica y científica internacional y garantizar un acceso equitativo a esos datos. Con tal sistema debería tratarse de garantizar que la parte que reciba los datos los proteja adecuadamente con arreglo a los principios enunciados en esta Declaración.

b)Los Estados deberían hacer todo lo posible, teniendo debidamente en cuenta los principios establecidos en la presente Declaración, para seguir fomentando la difusión internacional de conocimientos científicos sobre los datos genéticos humanos y los datos proteómicos humanos, favoreciendo a este respecto la cooperación científica y cultural, en particular entre países industrializados y países en desarrollo.

c)Los investigadores deberían esforzarse por establecer relaciones de cooperación basadas en el respeto mutuo en materia científica y ética y, a reserva de lo dispuesto en el Artículo 14, deberían alentar la libre circulación de datos genéticos humanos y datos proteómicos humanos con objeto de fomentar el intercambio de conocimientos científicos, siempre y cuando las partes interesadas observen los principios enunciados en esta Declaración. Con tal propósito, deberían esforzarse también por publicar cuando corresponda los resultados de sus investigaciones".

Art. 23 de la Declaración Internacional de la UNESCO de datos genéticos humanos: "Aplicación

b) En el contexto de la cooperación internacional, los Estados deberían esforzarse por llegar a acuerdos bilaterales y multilaterales que permitan a los países en desarrollo generar la capacidad necesaria para participar 


\section{aprovechamiento compartido de los beneficios ${ }^{26}$, pero también otras que} salvaguardan el respeto a los derechos humanos de la persona.

en la creación y el intercambio de saber científico sobre los datos genéticos humanos y de las correspondientes competencias técnicas".

${ }^{25}$ Entre los Códigos éticos y Protocolos relativos al uso compartido de datos podemos citar:

- Constitución de la Organización Mundial de la Salud (OMS 1946)

- Principios de Bermudas sobre secuenciación del genoma humano (1996)

- Declaración Universal sobre el Genoma Humano y los Derechos Humanos (UNESCO 1997)

- Convención de Derechos Humanos y Biomedicina (Consejo de Europa 1997)

- Declaración sobre muestra de ADN: control y acceso (HUGO 1998)

- Declaración sobre bases de datos genómicas humanas (Comité de Ética del HUGO 2002)

- Declaración sobre las consideraciones éticas de las bases de datos de salud (AMM 2002)

- Pautas éticas internacionales para la investigación biomédica en seres humanos (CIOMS, OMS 2002)

- Iniciativa de Budapest para el acceso abierto (2002)

- Uso compartido de datos de proyectos de investigación biológicos a gran escala: un sistema de responsabilidad tripartita (Declaración de Fort Lauderdale, 2003)

- Declaración Internacional sobre los Datos Genéticos Humanos (UNESCO, CIB 2003)

- Sociedad Europea de Genética Humana: almacenamiento de datos y bancos de ADN con fines de investigación biomédica (SEGH 2003)

- Declaración Internacional sobre Bioética y Derechos Humanos (UNESCO 2005)

- Protocolo Adicional de la Convención de Derechos Humanos y Biomedicina en relación a la Investigación Biomédica (Consejo de Europa 2005)

- Recomendación Rec (2006) 4 del Comité de Ministros a los Estados Miembros, sobre investigación con material biológico de origen humano (Consejo de Europa 2006)

- Principios y directrices de la OCDE sobre el acceso a los datos de la investigación financiada con fondos públicos (OCDE 2007)

- Pautas éticas internacionales para estudios epidemiológicos (CIOMS, OMS 2008)

- Recomendaciones de la cumbre internacional de 2008 sobre divulgación de datos proteómicos y políticas de uso compartido (Principios de Amsterdam, 2008)

- Directrices para los biobancos humanos y las bases de datos de investigación genéticas (OCDE 2008. 2009)

- Declaración de Toronto sobre uso compartido de datos en prepublicación (2009).

- Declaración conjunta de financiadores de investigación médica (2011).

- Buenas prácticas para biorepositorios: obtención, almacenamiento, recuperación y distribución de materiales biológicos para investigación ISBER 2012).

- Conducta responsable en la iniciativa de investigación global: un informe sobre políticas de actuación (InterAcademy Council 2012).

- Declaración de Helsinki (AMM 2013).

- Directrices sobre la protección de la privacidad y flujos transfronterizos de datos personales (OCDE 2013).

-Marco de actuación para el uso compartido responsable de datos genómicos y relativos a la salud (2014) del Global Alliance for Genomics and Health.

${ }^{26}$ Art. 19 de la Declaración Internacional de la UNESCO de datos genéticos humanos: Aprovechamiento compartido de los beneficios

“a) Los beneficios resultantes de la utilización de datos genéticos humanos, datos proteómicos humanos o muestras biológicas obtenidos con fines de investigación médica y científica deberían ser compartidos con la sociedad en su conjunto y con la comunidad internacional, de conformidad con la legislación o la política internas y con los acuerdos internacionales. Los beneficios que deriven de la aplicación de este principio podrán revestir las siguientes

formas:

i) asistencia especial a las personas y los grupos que hayan tomado parte en la investigación;

ii) acceso a la atención médica;

iii) nuevos diagnósticos, instalaciones y servicios para dispensar nuevos tratamientos o medicamentos obtenidos gracias a la investigación;

iv) apoyo a los servicios de salud;

v) instalaciones y servicios destinados a reforzar las capacidades de investigación;

vi) incremento y fortalecimiento de la capacidad de los países en desarrollo de obtener y tratar datos genéticos humanos, tomando en consideración sus problemas específicos;

vii) cualquier otra forma compatible con los principios enunciados en esta Declaración.

b) El Derecho interno y los Acuerdos internacionales podrían fijar limitaciones a este respecto". 
En relación a la cuestión planteada en estas páginas, son precisos compromisos institucionales (no individuales de cada investigador) y de nivel internacional que garanticen un uso eficiente y seguro de la información genética, si bien ello no resulta nada fácil, dada la complejidad ideológica, política, jurídica y cultural del Mundo.

En el orden internacional, creemos necesaria una norma explícita (estimamos que es posible la aplicación por analogía de alguna ya existente) que contemple:

> Un Estándar de Protección de los Derechos Fundamentales;

> Un Protocolo Marco de Actuación en el acceso y uso de información genética;

Un protocolo de consentimiento informado estandarizado de cesión de los datos genómicos;

$>\quad$ Condiciones de acceso; ¿público 0 de libre acceso (con datos anonimizados) o restringido con protocolos estrictos supeditados a Comités de acceso de datos (el usuario deba presentar su currículum y acreditar oportunamente que trabaja para una organización sin ánimo de lucro)?;

$>$ Comunicación de los resultados a los individuos y/o a los grupos;

$>$ Procedimientos para poner fin al uso compartido de datos;

$>\quad$ Establecer medidas de seguridad proporcionadas que reduzcan el riesgo de un acceso no autorizado, la pérdida de los datos y su uso indebido;

Sanciones.

A nivel nacional, ¿necesitamos una Ley de no discriminación por características genéticas ${ }^{27}$ A nuestro entender, creemos que el art. $14 \mathrm{CE}$, si bien no hace expresa mención a dicho tipo de discriminación, la cual tendría cabida en su inciso final ("los españoles son iguales ante la ley, sin que pueda prevalecer discriminación alguna por razón de nacimiento, raza, sexo, religión, opinión o cualquier otra condición o circunstancia personal o social') no la permite, por tanto, no es, en principio, necesaria. Asimismo, en la discriminación genética inter privatos, podríamos hablar

\footnotetext{
27 La Ley de 21 de mayo de 2008 de no discriminación por información genética (Genetic Information Nondiscrimination Act o GINA, en inglés) es una ley de Estados Unidos diseñada para prohibir el uso inapropiado de la información genética en seguro médico y el empleo.
} 
de la eficacia horizontal de los derechos fundamentals o Drittwirkung der Grundrech ${ }^{28}$.

¿Y una Ley de Garantías del patrimonio genético humano? Tal vez.

\section{A Modo de Conclusión}

Lo cierto es que nuestra vida genética y la de otras personas sea transparente y accesible a cualquiera causa vértigo.

La respuesta a los innumerables interrogantes en esta compleja materia pasa, a nuestro modo de ver, de un lado, por ser extremadamente cautelosos y elevar el nivel de protección de los derechos y libertades fundamentales; de otro, por generar confianza en el ciudadano hacia la comunidad científica acerca del valor del uso compartido de datos genómicos ${ }^{29}$.

\section{Referencias Bibliograficas}

Azzini S. Biobanche, Consenso e fonti del Diritto: un caso si eccezionale disordine, en La disciplina delle biobanche ai fini terapeutici e di ricerca, en CASONATO, Piciocchi y Varonesi, 2012, Università di Trento, p. 117-150. [Acceso en 2 jun 2015]

Disponible en: http://eprints.biblio.unitn.it/3979/1/casonato piciocchi veronesi quaderno 101 versio ne corretta giov19apr2012.pdf.

Battistuzzi F, Bonsignor L, Ciliberti A, De Stefano R. La condivisione genetica: una relazione pericolosa? II trattamento dei dati genetici del defunto nella realtà italiana. Generalità ed esemplificazione casistica. Rivista Italiana di Medicina Legale e del Diritto in campo sanitário n. 1: 59-67. [Internet]. 2014 [Acceso em 2 jun 2015] Disponible en:

\footnotetext{
${ }^{28}$ VIVAS-TESÓN, I.: "La horizontalidad de los derechos fundamentales", en Bienes de la personalidad. XIII Jornadas de la Asociación de Profesores de Derecho civil, Servicio de Publicaciones de la Universidad de Murcia, 2008, págs. 205-213.

29 Subrayan la importancia de la fiducia como elemento fundamental para el correcto funcionamiento de los biobancos MACILOTTI/IZZO/PASCUZZI/BARBARESCHI: "La disciplina giuridica delle biobanche", cit., pág. 99, la cual debe existir entre los tres actores en juego: donantes, gestores de biobancos e investigadores. Los citados autores subrayan que la relación de confianza que debe existir no sólo ha de ser jurídica sino también extrajurídica: "il Diritto infatti interviene la maggior parte delle volte a celebrare il momento patologico del rapporto di fiducia intercorrente tra i cittadini e la Scienza, ossia quando il rapporto di fiducia viene ad incrinarsi. Ma è necesario che il mondo della Sciencia medica si incarichi di alimentare tale rapporto, ponendosi come primario il problema della communicazione della Scienza ai cittadini. "Communicare correttamente la Scienza" è un ingrediente fondamentale perchè i cittadini decidano di donare i loro materiali biologici e di offrire i loro dati genetici alla ricerca. Tale operazione richiede uno sforzo in termini di adeguamento linguistico, di trasparenza, di capacità di ascolto, di capacità di auto legittimazione che rappresenterà il vero motore della espansione delle biobanks.
} 
http://www.iusexplorer.it/Rivista/Rivista Italiana di Medicina Legale/LA CONDIVISI ONE GENETICA UNA RELAZ?IdDatabanks=144\&ldDocMaster=4139832.

CABALLERO NAVARRO, T. M. Investigación biomédica, tratamiento de muestras genéticas humanas y biobancos. Revista Bioderecho.es [Internet]. 2014 [Acceso em 2.jun.2015...], n. 1: 1-15. Disponible en http://revistas.um.es/bioderecho/article/view/209371.

CAMBRON INFANTE, A. Los principios de protección de datos genéticos y la declaración de la UNESCO. Anuario da Facultade de Dereito da Universidade da Coruña, 2004, n. 8, p. 209-222. Disponible en: http://portal.unesco.org/es/ev.phpURL ID=17720\&URL DO=DO TOPIC\&URL SECTION=201.html. [Acceso em 2.jun.2015]

CÁRCAR BENITO, J.E. Internet, genética y Bioderecho. Una aproximación a las pruebas genéticas por internet: su proliferación, cese y regulación. Revista Bioderecho.es [Internet]. 2014 [Acceso en 2.jun.2015], 1(1):1-20. Disponible en http://revistas.um.es/bioderecho/article/view/209291/167191.

FERIOLI, E.; PICOZZI, M. La conservazione del materiale biologico finalizzato alla ricerca scientifica: questioni giuridiche e riflessioni etiche sulle biobanche. Rivista Medicina e Morale [Internet]. 2011 [Acceso en.2.jun.2015], 4(2): 553-584. Disponible en https://irinsubria.uninsubria.it/handle/11383/1731393\#.VbY 5vIVhBc.

MACILOTTI, M.; IZZO, U.; PASCUZZI, G.; BARBARESCHI, M. La disciplina giuridica delle biobanche. Pathologica [Internet]. 2008 [Acceso en.11.jun.2012], 100:86-101. Disponible en http://www.tissuebank.it/publicazioni/Macilotti.pdf.

PACIA, R. Ricerca genetica, biobanche e consenso informato. Famiglia e Diritto, Italia, 2012, 8 (9):838-852.

Campione Biologico e consenso informato nella ricerca genetica: il possibile ruolo delle biobanche. Jus Civile. Italia, p. 65-104, 2014. Disponible en: http://www.juscivile.it/contributi/2014/05 Pacia.pdf. [Acceso em 13.abr.2015]

SULEKOVÀ, M. Le biobanche di ricerca. Problematiche ético-sociali tra diritti dell"individuo e prospettiva comunitária. Rivista Medicina e Morale [Internet]. 2013 [Acceso en.12.jun.2015], 63(3): 477-509. Disponible en: http://www.biblio.liuc.it/scripts/essper/ricerca.asp?tipo=scheda\&codice $=11266843$.

TALLACCHINI, M. Dalle biobanche ai Genetic Social Networks. Immaginari giuridici e regolazione di materiali biologici e informazioni. Materiali per una storia della cultura giuridica, Italia, v.1, p. 157-182, 2013. Disponible en:

http://www.rivisteweb.it/doi/10.1436/73124. [Acceso en 30.mayo.2015]

Vivas-Tesón I. La horizontalidad de los derechos fundamentales. Bienes de la personalidad. XIII Jornadas de la Asociación de Profesores de Derecho civil, 2006, 
Santander. Artigo. Servicio de Publicaciones de la Universidad de Murcia, 2008, p. 205-

213. Disponible en: http://dialnet.unirioja.es/servlet/articulo?codigo=2996866. [Acceso em 30.mayo.2015]

Vivas-Tesón I. Bioinvestigación, biobancos y consentimiento informado del sujeto fuente vulnerable. Diario La Ley, no 7911, de 27 de julio 2012, p. 1-12. Disponible en: http://dialnet.unirioja.es/servlet/articulo?codigo=3977884. [Acceso en 30.mayo.2015]

Vivas-Tesón I. Bioresearch, biobanks and informed consent from vulnerable donors in Spanish Law. Europa e Diritto Privato, Italia, fasc. 4. p.1069-1095. Disponible en: http://papers.ssrn.com/sol3/papers.cfm?abstract id=2535056. [Acceso en 12.jun.2015]

Vivas-Tesón I. Ricerca biologica, biobanche e consenso informato del soggetto fonte vulnerabile nel Diritto Spagnolo. Rivista Critica del Diritto Privato [Internet]. 2014 [Acceso en 30.mayo.2015], n. 1: 105-136. Disponible en http://dialnet.unirioja.es/servlet/articulo?codigo=4793962.

Vivas-Tesón I. Consentimiento del adulto frágil al tratamiento de muestras biológicas y datos genéticos con fines de investigación biomédica: comparación entre el Derecho Español e Italiano. Revista de Derecho y Genoma Humano [internet]. 2014 [Acceso en.29.mayo.2015], 40: 95-130. Disponible en http://dialnet.unirioja.es/servlet/articulo?codigo $=4870063$. 